\title{
Adolescente renal crónico: alteraciones físicas, sociales y emocionales pos-trasplante
}

\author{
Ana Luisa Brandão de Carvalho Lira* \# \\ Maria Vilaní Cavalcante Guedes**/*** \# \#\# \\ Marcos Venícios de Oliveira Lopes***/**** \#
}

\author{
*Alumna del curso de Master en Enfermería \\ **Alumna del curso de Doctorado \\ ***Profesor de Enfermería \\ ****Doctor en Enfermería \\ \#Universidad Federal de Ceará. Fortaleza. Brasil \\ \#\#Universidad Estadual de Ceará. Fortaleza. Brasil
}

\section{RESUMEN}

La experiencia del trasplante renal en la adolescencia produce una sensación de incertidumbre del pronóstico, pero también de esperanza y de fe de encontrar estímulos para vencer los obstáculos. Realizado en el ambulatorio de trasplante renal de un hospital público es un estudio cualitativo, descriptivo cuyo objetivo es conocer las alteraciones físicas, sociales y emocionales en la vida del adolescente pos-trasplante renal. Doce adolescentes contestaron a las preguntas de una entrevista estructurada. Los resultados muestran que las limitaciones físicas más significativas fueron el alejamiento escolar, restricciones en la vida social y dificultad en practicar ejercicio. En relación a las alteraciones sociales, el ocio y la frecuencia escolar eran menores en los tres primeros meses pos-trasplante, período considerado más vulnerable. El apoyo de la familia y de los amigos fue el punto de soporte emocional identificado por los entrevistados. Los adolescentes adquirieron nuevas cualidades psicológicas para superar los obstáculos surgidos después lo trasplante.

PALABRAS CLAVE: TRASPLANTE RENAL ADOLESCENTE

CONDUCTA DE SALUD

\section{CHRONIC ADOLESCENT RENAL PATIENTS: PHYSICAL, SOCIAL AND EMOTIONAL POST- TRANSPLANT ALTERATIONS}

\section{SUMMARY}

Undergoing a kidney transplant in adolescence produces a feeling of uncertainty as to the prognosis, but also of hope and trust in finding stimuli to overcome obstacles. Conducted in the kidney transplant centre of a public hospital, it is a qualitative, descriptive study the aim of which is to determine the physical, social and emotional alterations in the life of a post-kidney transplant adolescent. Twelve adolescents replied to the questions in a structured interview. The results show that the most significant physical limitations were being away from school, restrictions in social life and difficulty in getting exercise. In relation to social alterations, leisure and going to school were lower in the first three months after the transplant, the period considered the most vulnerable. The support of family and friends was the point of emotional support identified by interviewees. Adolescents acquired new psychological qualities to overcome the obstacles arising after the transplant.

KEY WORDS: RENAL TRANSPLANT

ADOLESCENT

HEALTH BEHAVIOUR
Correspondencia:

Ana Luisa Brandão de Carvalho Lira

C/ Joaquim Nabuco, 275 / 801, Meireles

60125-120 Fortaleza (Brasil)

analuisabrandao@bol.com.br 


\section{INTRODUCCIÓN}

Brasil, de acuerdo con datos del Ministerio de la Salud, es el segundo país en el mundo en número de trasplantes renales, y aunque su crecimiento, entre 1997 y 2003, fue del 81,0\%, en febrero de 2004, existían 29.928 personas en lista de espera, de las cuales 6.256 eran de la región Nordeste (367 en el Estado de Cearáa $)^{(1)}$.

Aunque el trasplante renal proporciona una mejor calidad de vida siempre existe el riesgo de rechazo entre otras complicaciones, por lo que el paciente debe recibir información adecuada para que pueda adaptarse a su nueva forma de vi$\mathrm{da}^{(2)}$. Después de la realización del trasplante es fundamental el soporte al paciente, con el propósito de prever complicaciones, en especial el rechazo y las infecciones que puedan comprometer la supervivencia del injerto y del paciente $e^{(3)}$.

El éxito de un programa de trasplante renal no es solamente del equipo clínico y quirúrgico ya que también es importante la colaboración de otras especialidades, y de otros profesionales de salud, como psicólogos, asistentes sociales y biólogos ${ }^{(4)}$. La duración del soporte ambulatorio es indefinida y el trasplantado debe ser orientado sobre alimentación, higiene, medicación, ejercicios físicos, prevención de la infección; aclarando todas sus dudas.

Muchas veces crecer es una experiencia traumática, especialmente en la adolescencia, pues causa alteraciones físicas, sociales y emocionales. Las nuevas sensaciones ocasionan sentimientos negativos, como: carencia, miedo, inquietud y angustia; cuyos efectos pueden ser minimizados con el apoyo de la familia, de los amigos y de los profesionales de salud. La adolescencia es el período entre doce y veinte años de edad(5) que, en general, en los trasplantados se vuelve estresante, porque los hospitales no están estructurados para atender a los adolescentes, excepto en los grandes centros, ya que, en general, faltan profesionales entrenados específicamente para dar soporte a los adolescentes ${ }^{(6)}$.

En nuestra realidad, existen pocos estudios sobre el adolescente trasplantado renal. Sin embargo, es necesario conocer la dinámica del trasplante renal en la adolescencia, para conseguir una mejor calidad de vida del trasplantado y servir como orientación para los profesionales de salud.

Nuestro objetivo es conocer las alteraciones físicas, sociales y emocionales en la vida del adolescente renal crónico pos-trasplante.

\section{MATERIAL Y MÉTODO}

Es un estudio descriptivo, con análisis cualitativo. Se escogió este tipo de investigación porqué facilita conocer al grupo y sus características, describiendo con exactitud los hechos y fenómenos de la realidad escogida ${ }^{(7)}$.

La investigación fue realizada en el ambulatorio de trasplante renal de un hospital público, localizado en la ciudad de Fortaleza-CE, escogido por ser referencia en el Estado. La muestra la constituyen doce trasplantados renales.

Los criterios de inclusión fueron: ser adolescente entre 12 y 20 años, y haber sido trasplantado hace más de tres meses, pues se cree que en este intervalo surgen las primeras manifestaciones de respuestas psicosociales al trasplante. Los criterios de exclusión fueron: estar hospitalizado en el momento de la recogida de datos y no estar en condiciones físicas y/o mentales adecuadas para participar del estudio.

La recogida de datos se llevó a través de las historias clínicas y las entrevistas estructuradas realizadas en los días de las consultas de los adolescentes, con el fin de identificar los cambios que se habían producido en sus vidas después del trasplante. Todas las sesiones de entrevistas fueron grabadas con autorización de los informantes, garantizando su confidencialidad y la plena libertad para participar. Al ser los pacientes menores, la autorización se solicitó también a los padres o responsables, quiénes firmaron el consentimiento.

El período de recogida de los datos fue desde octubre de 2002 hasta marzo de 2003. Los entrevistados recibieron la letra A seguida por un número, con variación entre 1 y 12 , para preservar el carácter anónimo de sus identidades. Se inició la organización de los resultados con la trascripción de las entrevistas y, tras lecturas repetidas, se identificaron cuatro categorías cuyo análisis permitió definir la esencia del objeto en estudio.

El estudio fue aprobado por el Comité de Ética en Investigación en la institución hospitalaria, de acuerdo con las disposiciones de la Resolución 196/96, que define las directrices y normas de reglamentación de la investigación con seres humanos de Brasil(8).

\section{RESULTADOS Y DISCUSIÓN}

\section{Características del grupo}

De los doce adolescentes que constituyen la muestra seis son varones y seis mujeres, con edades entre doce y veinte años 
(media de 15,8 años). En cuanto la escolaridad, la mayoría $(83,3 \%)$ tenía hasta 8 años de estudios.

La renta familiar mensual de la mayoría $(58,3 \%)$ de los adolescentes era $\mathrm{R} \$ 260,00$ (unos $70 €$ ). Todos recibieron el riñón de donantes vivos relacionados (padre, madre, tíos y hermanos), siendo la mitad de los donantes la madre. El tiempo transcurrido tras el trasplante de la mayoría $(58,3 \%)$ fue de más de un año, con una media de tres años.

De los discursos de los participantes, tras ser discutidos e interpretados con apoyo en la literatura revisada, surgieron las siguientes temáticas.

Alteraciones físicas en la vida del adolescente renal crónico pos-trasplante

Al convivir con la enfermedad, el paciente renal crónico, aunque se perciba cómo una persona normal, reconoce algunas limitaciones relacionadas con alimentación, ingestión hídrica $e$ implicaciones en la utilización de su tiempo y en sus actividades de ocio ${ }^{(9)}$.

Con relación a la alimentación de los adolescentes estudiados, la mayoría $(66,7 \%)$ sigue una dieta pobre en grasas e hiposódica, evitando tomar, dentro y fuera de casa, alimentos como patatas fritas, salados y enlatados. El estado nutricional del paciente renal crónico es uno de los factores pronósticos de mayor morbi-mortalidad ${ }^{(4)}$ por tanto, los pacientes trasplantados deben tener una posición participante en su tratamiento, principalmente en lo que se refiere a la alimentación; además la persona trasplantada tendrá que ser muy cuidadosa con la higiene y con los ambientes frecuentados.

Otro aspecto importante es que tendrá que tomar inmunosupresores de por vida ${ }^{(4)}$. En el pos-trasplante, la terapia imunosupresora y el acto quirúrgico provocan efectos metabólicos tales como hipercatabolismo proteico, ocasionando la cicatrización lenta de la herida, susceptibilidad a la infección, y obesidad, que es atribuida a la mejora del bienestar después lo trasplante y al uso de glicocorticoides ${ }^{(4)}$. Esto afecta la imagen corporal de los adolescentes, causando, muchas veces, sentimientos negativos sobre características y funciones de su cuerpo. Entre los comportamientos más observados referentes a la alteración de la imagen corporal, tenemos expresión de sentimientos negativos sobre el cuerpo, de preocupación con los cambios y de sentimiento de vergüenza. Se puede observar estos comportamientos en las frases siguientes:

- "Tuve miedo de engordar. Yo no tomo aquel medicamento que tiene corticoide. Los dos que tomo no hay aquí, pero así mismo yo tuve miedo de engordar, de se me poner fea". (A7)
- "Yo me quedé con ésas tres cicatrices. Cuando yo voy a playa, tengo vergüenza de andar mostrando la barriga". (A12)

Podemos identificar en los relatos sentimientos negativos sobre el cuerpo, principalmente en relación al miedo a engordar y la vergüenza de la cicatriz. Lamentablemente, uno de los efectos experimentados por casi todas las personas que toman corticosteroides es un gran aumento del apetito ${ }^{(10)}$. Por tanto, el aumento de peso es, un serio problema a largo plazo, siendo necesaria la dieta con poca grasa y azúcar.

Los sentimientos vividos por los adolescentes entrevistados de anormalidad y de no seguir patrones sociales comúnmente aceptados les hacen sentirse diferentes, la comunicación se hace difícil, llevándolos muchas veces, al aislamiento de la sociedad. En cuanto a las limitaciones físicas consecuentes a la cirugía, las más significantes se referían a la interrupción de los estudios escolares, restricciones en la vida social, dificultad de practicar ejercicios físicos y se expresan en los relatos:

- "Paré de estudiar, pues estaba aturullando. Venía hacia el hospital y se quedaba perdiendo clase". (A3)

- "Con relación la fiesta, siempre tiene hora cierta para llegar a casa por mi causa". (A2)

- "Yo a veces juego. Solo que yo no echo mucho, porque puede llevar algún golpe en el riñón". (A5)

Esas limitaciones, unidas a las consecuencias económicas y sociales, agravan aún más el cuadro de alteración de la imagen corporal, a medida que los adolescentes se ven incapaces de realizar actividades consideradas comunes y simples para su edad.

Alteraciones sociales en la vida del adolescente renal crónico pos-trasplante

A pesar de las limitaciones pos-trasplante, verificamos que los adolescentes lograron mantener alguna forma de ocio. Entre las opciones de ocio en la fase inicial pos-trasplante, la mayoría $(58,3 \%)$ resaltó la charla con los amigos, pues durante los primeros meses después la cirugía, período considerado más vulnerable, los pacientes evitan exponerse a ambientes aglomerados, como el cine.

Después ese período de mayor vulnerabilidad, los adolescentes se sienten más seguros y buscan otras formas de ocio, como jugar con amigos y familiares, andar en bicicleta, leer, bailar y enamorarse. Por medio del ocio, los adolescentes buscaron superar sus dificultades, incorporarse al contexto social, volviendo así a una vida más normal, al lado de sus amigos y familiares. 
Con relación a la escuela, el papel de estudiante se alteraba en el período inicial, debido las limitaciones y cuidados pos-trasplante; los adolescentes dicen:

- "Lo trasplante interfirió solamente cuando yo fui para la escuela. Fui de máscara, ahí el personal preguntó si yo tenía problema de garganta". (A10)

En nuestro estudio, el alejamiento escolar fue más común en los primeros meses después del trasplante, período en el cual el paciente debe comparecer diariamente al ambulatorio de trasplante renal para tratamiento. La oportunidad de volver a estudiar aumenta después de ese período, cuando la frecuencia a las consultas ambulatorias disminuye y pasan a ser mensuales.

Sin embargo, observamos que la frecuencia escolar es aún menor en el período de hemodiálisis, como observamos en las declaraciones:

- "Perdí un año en la escuela, pero fue antes del trasplante, a causa de la hemodiálisis". (A8)

- "Me quedé dos años sin estudiar, a causa del problema en el riñón, porque yo vivo lejos y tenía que venir tres veces en la semana; ahí no daba para estudiar y venir acá". (A3)

Generalmente la enfermedad surge de forma repentina $e$ intensa; aunque el adolescente mejore, muchas veces se queda apartado de la escuela, por el tratamiento, debido a las sesiones de hemodiálisis antes del trasplante y a las limitaciones y cuidados en el pos-trasplante inmediato.

Alteraciones emocionales en la vida del adolescente renal crónico pos-trasplante

El punto de soporte emocional importante identificado por los adolescentes entrevistados, fue el apoyo ofrecido por la familia y por los amigos. Cuando se percibían protegidos, sus sentimientos de seguridad y esperanza en el porvenir se reforzaban y se percibían amparados y comprendidos. Esto seguramente facilitó una mayor adaptación, ofreciéndoles, incluso, mecanismos para enfrentarse a los obstáculos de una manera más efectiva. En estas declaraciones, se percibe el significado de ese apoyo:

- "Mi madre acompaña todas mis cosas. Mi tía, mi familia, a pesar de ellos no vivan aquí, llaman y preguntan cómo es que estoy, si yo puedo ir". (A2)

- "Con mis amigos es normal. Preguntan cómo es que fue el examen, como es que fue hecho, ahí yo contesto lo que ellos quieren". (A4)

El hecho de recibir apoyo de las personas queridas permite al adolescente sentirse mejor, pues tiene la seguridad de la pro- ximidad de los familiares y de los amigos compartiendo con ellos tanto momentos alegres como tristes. Las personas en estrecha convivencia con el enfermo tienen un papel muy importante, pues, muchas veces es el miedo de ser rechazado por el otro lo que impide a muchos que se consideren como seres coexistentes con otros seres ${ }^{(9)}$.

Otro cambio percibido fue la adquisición por los adolescentes de nuevas cualidades psicológicas a la medida que se veían capaces de enfrentarse a situaciones de extremo estrés físico, social y emocional. Eso les permitió percibir la posibilidad de superar los obstáculos considerados infranqueables. En los relatos a continuación, se puede observar:

- "Tenemos que pensar primero en lo que estamos cargando adentro de nosotros, porque es como me dijeron, eso es un cuerpo extraño y cualquier cosa que pase yo pierdo el riñón." (A12)

- "Antiguamente, era muy irritada con todo, hoy yo estoy mejor". (A7)

El trasplante, por tanto, posibilitó a los adolescentes reflejar sobre su modo de sentir y actuar delante de situaciones difíciles, permitiéndoles una mayor autoestima, al mejorar la percepción de sí mismo como una persona más fuerte y capaz de superar tal experiencia de una manera digna y satisfactoria.

\section{Consideraciones Finales}

Con ese estudio, se puede aprehender que el trasplante renal causa gran trastorno en la vida del adolescente y de aquéllos que le rodean. La experiencia del trasplante renal en la adolescencia es una sensación de incertidumbre del pronóstico, pero también de esperanza y de fe en encontrar estímulos para vencer los obstáculos. Las limitaciones impuestas a los adolescentes después del trasplante son inferiores al período en que estaban en hemodiálisis. Las alteraciones físicas más significativas fueron la dieta, los disturbios en la imagen corporal, el alejamiento escolar y la dificultad para practicar ejercicios.

En cuanto a las alteraciones sociales, percibimos que el ocio y la frecuencia escolar eran menores en el postoperatorio inmediato, debido a los cuidados necesarios para evitar complicaciones, como el rechazo.

Con relación a las alteraciones emocionales, percibimos la importancia del apoyo de la familia y de los amigos para el enfrentamiento positivo y para la búsqueda de medios que mejoren las condiciones de vida. La vivencia del trasplante renal, a pesar de lo difícil de la situación, les permitió la adquisición de nuevas cualidades psicológicas. La vida pasó a ser más valorada y ellos se apropiaron del sentimiento de mayor responsabilidad por el cuidado y mantenimiento de su salud. 


\section{BIBLIOGRAFÍA}

1. Brasil. Ministério da Saúde. Dados sobre transplante renal. Disponible en: http://dtr2001.saude.gov.br. Accedido en 16 mayo 2004.

2. Mendes CA, Shiratori K. As percepções dos pacientes de transplante renal. Rev Nursing 2002; 5(44):15-22.

3. Sampaio FPR, Pagliuca LMF. O transplante renal em acompanhamento ambulatorial: autocuidado higiênico-dietético e medicamentoso. Fortaleza: FCPC; 2000.

4. Barros E, Manfro RC, Thomé FS, Gonçalves LFS. Nefrologia: rotina, diagnóstico e tratamento. Porto Alegre: Artmed; 1999.

5. Wong DL. Enfermagem pediátrica: elementos essenciais à intervenção efetiva. Rio de Janeiro: Guanabara Koogan; 1999.
6. Campos TCP. Psicologia hospitalar: a atuação do psicólogo em hospitais. São Paulo: EPU; 1995.

7. Triviños ANS. Introdução à pesquisa em ciências sociais: a pesquisa qualitativa em educação. São Paulo: Atlas; 1987.

8. Brasil. Conselho Nacional de Saúde. Resolução no 196/96. Decreto no 93.933 de Janeiro de 1987. Estabelece critérios sobre pesquisas envolvendo seres humanos. Brasília: O conselho; 1996.

9. Barbosa JC, Aguillar OM, Boemer MR. O Significado de conviver com a insuficiência renal crônica. Rev Bras Enferm 1999; 52 (2):293-302.

10. Piveta V. Manual de orientação para o paciente transplantado renal. São Paulo: Hospital das clínicas; 1999. 